\title{
Carnets
}

Revue électronique d'études françaises de l'APEF

Première Série - 2 Numéro Spécial | 2010

Littératures nationales: suite ou fin. Résistances, mutations \& lignes de fuite

\section{L'universel et le particulier : enjeux et présupposes de la "littérature-monde" en français}

Nicolas di Meo

\section{(2) OpenEdition}

Journals

Édition électronique

URL : http://journals.openedition.org/carnets/4916

DOI : $10.4000 /$ carnets.4916

ISSN : 1646-7698

Éditeur

APEF

Édition imprimée

Date de publication : 1 juin 2010

Pagination : 55- 68

Référence électronique

Nicolas di Meo, "L'universel et le particulier : enjeux et présupposes de la "littérature-monde" en français », Carnets [En ligne], Première Série - 2 Numéro Spécial | 2010, mis en ligne le 16 juin 2018, consulté le 19 avril 2019. URL : http://journals.openedition.org/carnets/4916 ; DOI : 10.4000/ carnets. 4916

Carnets est mis à disposition selon les termes de la licence Creative Commons - Atribution - Pas d'utilisation commerciale 4.0 International. 


\section{L'UNIVERSEL ET LE PARTICULIER: ENJEUX ET PRESUPPOSES DE LA “LITTERATURE-MONDE” EN FRANÇAIS}

NICOLAS DI MEO

Université Michel de Montaigne Bordeaux 3

nicolas_dimeo@hotmail.com

\section{Résumé}

Paru au printemps 2007, l'ouvrage collectif Pour une littérature-monde fait suite au manifeste "Pour une littérature-monde en français", publié dans Le Monde du 16 mars 2007. Les deux textes condamnent le nationalisme culturel français et critiquent l'idée de littérature nationale, fondée sur la croyance en un rapport privilégié entre une langue et une nation. Ils défendent la conception d'un universel dépendant non de la conformité à un ensemble de règles fixées de manière arbitraire, mais d'une expérience du local, du particulier, qui serait la condition de l'accès au monde dans sa globalité et sa complexité. Malgré des différences notables, cette vision des choses n'est toutefois pas complètement étrangère à l'histoire intellectuelle et littéraire qu'elle dénonce: la dialectique de l'universel et du particulier occupait déjà une place importante dans le champ littéraire français du début du $X X^{e}$ siècle. Ce sont cependant les usages politiques de ce discours qui ont changé, dans la mesure où il ne s'agit plus de vanter la France dans une perspective mêlant nationalisme et cosmopolitisme, mais de lutter contre les ravages de l'impérialisme culturel français.

\section{Abstract}

On March $16^{\text {th }}$, 2007 a manifesto entitled "Pour une littérature-monde en français" ("Toward a WorldLiterature in French") was published in the newspaper Le Monde. It was followed two months later by a collective book called Pour une littérature-monde. Both texts condemned French cultural nationalism (as well as the idea of French universalism) and criticized the notion of national literature, because this concept suggests the existence of a privileged relationship between one language (French) and one nation (France). According to most contributors to the book, only the experience of the local can lead to the universal. This idea, however, is not a new one. It was already a common representation in the first half of the $20^{\text {th }}$ century. But in spite of this apparent continuity, huge differences remain, since the words "local", "universal", and "identity" have acquired new meanings. In terms of politics, Pour une littérature-monde uses the local/universal dialectic in various strategies aimed at denouncing French cultural imperialism.

Mots-clés: diversité, francophone, identité, littérature-monde, local, universel

Keywords: diversity, Francophone, identity, local, universal, world-literature 
Publié au printemps 2007, l'ouvrage collectif Pour une littérature-monde, dirigé par les écrivains Jean Rouaud et Michel Le Bris, fait suite au manifeste "Pour une littératuremonde en français" (également connu sous le nom de "Manifeste des 44"), paru dans le quotidien Le Monde du 16 mars 2007. Dans un contexte de campagne électorale caractérisé par des débats virulents autour des idées de nation et d'identité française, les deux textes ne passent pas inaperçus ${ }^{1}$. Leurs enjeux se situent dans la remise en cause du lien privilégié entre la nation et la langue françaises, dans la condamnation des prétentions universalistes de la littérature française et dans la critique du champ littéraire français, jugé nombriliste et sclérosé. Deux reproches principaux sont adressés aux intellectuels et aux écrivains français: d'une part, celui de vivre dans un milieu étroit, essentiellement parisien, et de ne pas accorder suffisamment de crédit aux auteurs venus d'ailleurs; d'autre part, celui de ne se préoccuper que de jeux formels et de tourner le dos au monde "réel", avec ses problèmes, ses mutations et ses défis. Les dimensions esthétique et politique sont imbriquées et la création littéraire digne de ce nom est présentée par les divers contributeurs comme un moyen de comprendre les sociétés contemporaines, faites d'hybridations et de mélanges, tout en subvertissant les rapports de domination les plus injustes et les plus persistants.

Pour une littérature-monde contient donc une critique du nationalisme littéraire, mais aussi une critique de l'idée même de littérature nationale. Celle-ci est accusée d'être au service de l'impérialisme culturel français et de favoriser un classement arbitraire des écrivains francophones en fonction de leur pays d'origine, dans une logique où les auteurs seraient cantonnés à une mission de représentation et auraient pour fonction d'exprimer l'identité de leur communauté, d'exposer ses problèmes, voire de perpétuer ses traditions, mais n'auraient pas vocation à s'intéresser à des questions plus globales, plus universelles, réservées aux seuls représentants du centre incontesté que constituerait la France métropolitaine. Ce qui est dénoncé, ici, c'est donc un cloisonnement du champ littéraire, mais aussi une restriction de la liberté créatrice des écrivains dits francophones. Pour remédier à cela, les différents signataires souhaitent la constitution d'un champ littéraire de langue française qui soit vraiment multiculturel. Selon Alain Mabanckou, les écrivains français sont en effet des écrivains francophones au même titre que les autres et doivent trouver leur place au sein d'un ensemble multinational à l'intérieur duquel ils n'ont ni plus ni moins de légitimité que leurs confrères (Mabanckou, 2007: 56). Nancy Huston, pour sa part, considère l'écriture comme la traduction d'un univers intérieur et refuse toute forme d'assignation identitaire, qu'elle soit régionale ou nationale: "[...] je fais mienne cette déclaration de Ying Chen, romancière québécoise d'origine chinoise installée en Californie:

\footnotetext{
${ }^{1}$ Le manifeste et l'ouvrage collectif ont suscité de très nombreux commentaires et comptes rendus, y compris de la part des candidats à l'élection présidentielle de 2007. Du côté des chercheurs, ces textes ont également suscité de nombreux travaux, à l'image du colloque Littérature-monde: New Wave or New Hype?, qui s'est tenu à l'Université de Floride (Florida State University), à Tallahassee, au mois de février 2009.
} 
'Si vous devez me mettre des étiquettes, de grâce, mettez-m'en le plus possible"' (Huston, 2007: 152). Les signataires revendiquent une liberté de création qui ne fasse pas de l'écrivain le représentant d'une nation ou d'une culture garantissant son authenticité. Le poète et romancier Nimrod se méfie ainsi des interprétations réduisant la littérature à l'expression d'une identité mythifiée:

Nous [les Africains] ne poursuivons jamais que la tradition littéraire occidentale. [...] Pour être authentiquement africaine, notre littérature devrait être écrite en nos langues. Constatons seulement que nous en sommes encore bien loin, heureusement. Car "l'authenticité" [...] accouche toujours des pires malheurs (Nimrod, 2007: 220).

Abdourahman A. Waberi, quant à lui, dénonce le rôle de gardiens des traditions que l'université française impose aux auteurs africains:

Les contenus et les figures littéraires renvoyant à la "tradition" - une catégorie inventée pour les besoins de la cause - sont portés au pinacle de telle sorte que les gardiens du temple puissent geindre à la mort de tout vieillard affublé d'une longue barbe et d'un boubou immaculé en débusquant dans les cendres de sa logorrhée les derniers sursauts d'une bibliothèque en fumée (Waberi, 2007: 69).

Ce passage est une attaque contre la célèbre formule d'Amadou Hampâté Bâ ("En Afrique, quand un vieillard meurt, c'est une bibliothèque qui brûle"), ou plutôt contre l'utilisation qui est faite de cette formule en Occident, où elle sert souvent à justifier la représentation stéréotypée d'une Afrique traditionnelle cultivant des traditions immémoriales sans accorder la moindre place à l'innovation individuelle. Ainsi, les auteurs des différents articles composant Pour une littérature-monde s'accordent sur un point capital: la littérature vivante et créatrice, la seule digne d'intérêt, est faite d'emprunts et d'hybridations, d'échanges et de rencontres à l'échelle internationale, si bien qu'en aucun cas le cadre de la nation ou de la tradition ne peut contenir l'univers d'un grand créateur.

Est-ce à dire, pour autant, qu'il n'existerait aucun lien entre appartenance identitaire et création littéraire? Les choses sont beaucoup plus complexes. Dans sa contribution, Alain Mabanckou, après avoir déploré la scission trop souvent observée entre littérature française et littératures francophones, ajoute la remarque suivante: "Jamais il ne sera question d'abandonner son être ou de le vendre aux enchères publiques. Je suis conscient et plus que convaincu que c'est en partant du 'local' qu'on atteint le monde, l'universel' (Mabanckou, 2007: 63). Son but est d'appeler les écrivains dits francophones à ne pas renier leur identité dans l'objectif d'être considérés comme des écrivains français, mais aussi, à l'inverse, à ne 
pas exagérer les marques de cette identité pour être lus comme des auteurs exotiques, autrement dit pour occuper une sorte de niche dans le marché littéraire. II s'agit, en d'autres termes, de remettre en cause un système de reconnaissance et de légitimation suivant lequel les écrivains qui ne sont pas originaires du centre du champ littéraire, c'est-à-dire de France métropolitaine, sont souvent incités à cultiver des différences stéréotypées correspondant moins à des réalités culturelles existant effectivement qu'à des attentes préconçues du marché et du lectorat français (Moura, 2010: 33). Ainsi, la dialectique du local et de l'universel que propose Alain Mabanckou oppose à l'universalisme que revendiquerait la tradition littéraire française une autre forme d'universalisme, moins rigide, moins abstraite, car trouvant sa source non dans des valeurs immuables, mais dans une expérience de la spécificité. Dans cette perspective, il s'agit moins de se conformer à des attentes ou à des canons prédéfinis que d'apporter sa contribution au trésor collectif de l'humanité en exprimant ce que l'on porte en soi d'unique et d'inimitable. Entre l'universalisme issu des Lumières, puis de l'européocentrisme ayant connu son apogée durant la période coloniale et cet universel d'un genre nouveau, tiré du particulier, le changement de paradigme semble complet: l'accent est mis sur la singularité et la spécificité, au détriment de la conformité à des modèles jugés valables pour tous. Ce qui est suggéré, ici, c'est donc une façon différente de penser l'articulation du local et du global, en accord avec une vision multiculturelle du monde.

Encore faut-il nuancer la nouveauté d'une telle approche. Opposer cette dialectique de l'universel et du particulier à un universalisme esthétique qui constituerait le dogme de la littérature française et n'accorderait aucune place aux différences demeure relativement simpliste. Certes, la défense d'une esthétique classique faite d'harmonie et de mesure a longtemps contribué à imposer l'idée selon laquelle il existerait des normes et des codes d'écriture auxquels tout auteur de qualité devrait se conformer. Mais le discours faisant du local ou du particulier la source de l'universel n'est pas nouveau pour autant. Dès la fin du $\mathrm{XIX}^{\mathrm{e}}$ siècle, il est au cœur des représentations esthétiques et identitaires de nombreux écrivains français. Dans Les Déracinés (1897), Maurice Barrès considère la fidélité aux traditions régionales comme la source d'un rapport harmonieux et apaisé à la patrie dans son ensemble, mais aussi, dans un second temps, comme le seul moyen d'accéder à la "raison internationale" sans devenir la victime d'une modernité uniforme et destructrice:

Dans une patrie, il faut ce point fixe: une conscience, non pas immuable, mais qui s'analyse et qui évolue, en ne perdant ni sa tradition, ni le sens de sa tradition. C'est un lieu national, où quelques privilégiés, délégués de chaque génération, viennent s'élever jusqu'à la raison internationale, humaine, en comprenant toutes les conditions de l'existence sous tous les climats, en comprenant que la dissemblance des visages 
nécessite celle des mœurs, comme l'éloignement des pays celui des sentiments (Barrès, 1994: 557).

L'universel, aux yeux de Barrès, ne se conçoit que dans l'expérience du divers. Sous des formes différentes, cette idée reste très répandue durant la première moitié du $X X^{e}$ siècle. Déplorant, en 1915, la destruction de la cathédrale de Reims par l'armée allemande, Romain Rolland estime que le caractère universel de ce monument vient du fait qu'il est l'expression la plus parfaite de la "race" française:

Une œuvre comme Reims est beaucoup plus qu'une vie: elle est un peuple [...]; elle est l'arbre de la race, dont les racines plongent au plus profond de sa terre et qui, d'un élan sublime, tend ses bras vers le ciel. Elle est bien plus encore: sa beauté qui domine les luttes des nations est l'harmonieuse réponse faite par le genre humain à l'énigme du monde, - cette lumière de l'esprit, plus nécessaire aux âmes que celle du soleil (Rolland, 1915: 10).

Romain Rolland articule deux dimensions apparemment contradictoires: d'un côté, la cathédrale de Reims est envisagée comme "l'arbre de la race" française, autrement dit comme une production à la fois mystique, esthétique et architecturale incarnant de manière presque parfaite la spécificité supposée des Français (le mot "race", très ambigu, n'est pas employé dans un sens exclusivement biologique, mais inclut aussi des éléments culturels, ce qui était fréquent à l'époque); d'un autre côté, la cathédrale est présentée comme "l'harmonieuse réponse faite par le genre humain à l'énigme du monde", donc comme une œuvre possédant un caractère universel indéniable, car susceptible de s'adresser au "genre humain" tout entier. En fait, les deux aspects, selon l'auteur, sont inséparables: la fidélité au "génie" d'une population (ou d'une "race") apparaît comme la condition permettant à l'œuvre de faire sens non seulement aux yeux de ceux qui l'ont produite et de leurs descendants, mais aussi auprès d'un public beaucoup plus large, qui reconnaît en elle une forme d'authenticité garante de sa signification et de son intérêt pour l'ensemble de l'humanité.

Ce lien entre déterminisme identitaire, authenticité et universalité est courant dans la première moitié du $X X^{e}$ siècle. André Gide, lui aussi, ne manque pas de se faire l'écho d'une telle dialectique de l'universel et du particulier. En 1909, dans un article intitulé "Nationalisme et littérature", il défend l'idée paradoxale selon laquelle les artistes les plus "généralement humain[s]“, c'est-à-dire les plus universels, les plus aptes à toucher un public mondial, seraient les dépositaires du "génie" de leur peuple ou de leur nation:

[...] [l]es œuvres les plus humaines, celles qui demeurent d'intérêt le plus général, sont aussi bien les plus particulières, celles où se manifeste le plus spécialement le 
génie d'une race à travers le génie d'un individu. Quoi de plus national qu'Eschyle, Dante, Shakespeare, Cervantès, Molière, Goethe, Ibsen, Dostoïevski? Quoi de plus généralement humain? Et aussi de plus individuel? (Gide, 1999: 177)

En 1936, dans son éloge funèbre de Maxime Gorki, prononcé sur la place Rouge à Moscou, il continue de s'appuyer sur cette thèse pour établir une équivalence entre les dimensions individuelle, nationale et universelle: "Aucun écrivain russe n'a été plus russe que Maxime Gorki. Aucun écrivain russe n'a été plus universellement écouté" (Gide, 2001: 788). La fidélité aux origines apparaît dans cette logique comme la meilleure garantie d'une audience universelle; l'écrivain qui ne rompt pas avec le "génie" de sa nation et exprime la personnalité intime de son peuple est aussi celui qui aura le plus de chance d'être écouté et respecté par les lecteurs du monde entier.

Même le discours sur l'universalisme supposé de la France et de sa littérature, qui constitue un lieu commun sous la Troisième République, est régulièrement rapporté à des causes identitaires. Les thèmes de l'unité dans la diversité, de l'aptitude à l'harmonie et à la synthèse, qui sont au cœur de la construction de l'identité française entre 1850 et 1950 (Thiesse, 1999: 192-193), sont régulièrement présentés comme les conséquences de la grande diversité des provinces françaises et de la nécessité d'unifier tant de régions dissemblables au sein d'une nation cohérente. Selon cette construction idéologique, dont les implications patriotiques sont évidentes, la France, en raison de sa diversité intérieure, possèderait une vocation au cosmopolitisme qui la distinguerait des autres pays (Di Méo, 2009: 117-124) et aurait plus qu'aucun autre le sens de l'universel: "Observez le paradoxe: avoir pour spécialité le sens de l'universel" (Valéry, 1960: 1058). Aux yeux d'un auteur comme Paul Valéry, c'est cette spécificité qui expliquerait les caractéristiques de la littérature française, au premier rang desquelles figureraient le classicisme, le sens de la forme et le goût de l'ironie. Ces propriétés supposées sont envisagées comme des réponses au problème constitutif de la nation, à ce besoin de créer de l'harmonie entre des influences multiples en prenant suffisamment de distance pour n'en privilégier aucune et en les assimilant au sein de productions esthétiques possédant une architecture parfaite: "[...] il n'est pas douteux [...] que la littérature de ce pays, en ce qu'elle a de plus caractéristique, procède [...] d'un mélange de qualités très différentes et d'origines très diverses, dans une forme d'autant plus nette et impérieuse que les substances qu'elle doit recevoir sont plus hétérogènes" (Valéry, 1960: 113). Le nationalisme de ces réflexions ne fait aucun doute; mais ce qui est intéressant, surtout, c'est le mode de pensée que révèle un tel raisonnement: la notion d'universalité est présentée comme le résultat d'une histoire, comme la conséquence d'une particularité de la nation française; elle dépend donc d'une construction identitaire censée la légitimer en la prémunissant contre toute accusation d'abstraction, dans 
un contexte valorisant fortement la fidélité au "génie" ou aux "caractères" nationaux, aussi stéréotypés soient-ils.

L'idée selon laquelle l'accès à l'universel passerait par le local ou le particulier n'est donc pas récente. Cela dit, les ressemblances apparentes ne doivent pas masquer de profondes différences. La dialectique de l'universel et du particulier telle qu'on l'observe chez Barrès, Gide ou Valéry ne sert pas à contester, mais à fonder le concept de littérature nationale, qu'Alain Mabanckou récuse au contraire. Qu'est-ce qui, par conséquent, a changé depuis le début du $\mathrm{XX} \mathrm{X}^{\mathrm{e}}$ siècle? L'évolution ne concerne pas l'idée selon laquelle l'accès à l'universel passerait par la fidélité de l'écrivain à l'identité qui est la sienne, c'est-à-dire à l'expérience spécifique du monde qui l'a façonné; sur ce point, la continuité est totale. C'est en revanche dans la façon de concevoir et de construire les identités elles-mêmes que s'observe une mutation décisive. II n'est plus question aujourd'hui du "génie d'une race" et l'identité n'est plus synonyme d'appartenance nationale. Ou tout au moins elle ne l'est plus exclusivement: les identités - au même titre que les cultures - sont de moins en moins perçues comme des substances et de plus en plus envisagées comme des constructions complexes, en perpétuelle évolution, intégrant des facteurs multiples (Appadurai, 2005: 4345). La remise en cause de la notion de littérature nationale est donc inséparable de la remise en question d'une vision restrictive et déterministe de l'appartenance nationale, ce qui a aussi pour conséquence d'abolir toute idée de hiérarchie entre les formes d'universalité, dans la mesure où il n'est plus pensable aujourd'hui, contrairement à ce qu'affirmaient de nombreux auteurs de la fin du $X X^{e}$ et de la première moitié du $X X^{e}$ siècles, de prétendre que l'universel français, en raison de la diversité extraordinaire de la nation, serait en quelque sorte plus universel que les autres. A l'origine du concept de littérature nationale se trouvait la croyance en l'existence d'une identité propre à chaque pays, dont tout citoyen aurait été le dépositaire et tout écrivain le porte-parole. Or aujourd'hui cette conception pose problème, comme on l'a vu récemment en France avec les réactions suscitées par le débat sur l'identité nationale ${ }^{2}$. De nombreux commentaires publiés dans la presse - qu'il s'agît d'articles d'opinion, d'analyses de chercheurs ou tout simplement de courriers de lecteurs se sont en effet plus attachés à contester les termes du débat, à en mettre au jour les enjeux, qu'à définir ce que serait une hypothétique identité nationale française.

II semble donc problématique de continuer à parler de littérature nationale, et surtout de donner un contenu précis à cette notion, puisque le substrat qui la fondait, c'est-à-dire le concept d'identité nationale, n'est plus considéré comme pertinent. Mais l'idée selon laquelle

\footnotetext{
2 Annoncé dès l'élection de Nicolas Sarkozy en mai 2007, officiellement lancé en novembre 2009 par Eric Besson, ministre de l'immigration, de l'intégration, de l'identité nationale et du développement solidaire, le débat sur l'identité nationale n'a pas reçu un accueil favorable dans la presse et la société françaises. Non seulement il a été perçu comme un instrument électoraliste, à quelques mois des élections régionales de mars 2010, mais en plus il a été régulièrement interprété comme une tentative de figer l'identité de la nation en en niant la diversité, la complexité et, surtout, l'évolution permanente.
} 
l'universel trouverait son origine dans le local ou le particulier ne disparaît pas pour autant. Alain Mabanckou n'est pas le seul, dans Pour une littérature-monde, à défendre cette thèse. Abdourahman A. Waberi fait ainsi l'éloge de ceux qu'il considère comme les "aînés" de la littérature-monde (Stevenson, Segalen, Glissant) et affirme que ces auteurs "conjuguent le mouvement et l'enracinement" (Waberi, 2007: 71). Gary Victor, pour sa part, condamne la notion de "littérature nationale": "Donner une nationalité au sens strict à la création, c'est la fossiliser, l'exclure de certains lieux et l'empêcher de déployer librement ses ailes" (Victor, 2007: 315). Mais cela ne l'empêche pas, à la fin de son article, d'articuler identité locale et universalité :

Dans le village global qui se construit souvent contre nous [les habitants du Sud], chaque lieu est forcé pour défendre son authenticité de partir à la découverte des autres lieux. Le travail du créateur est donc double. II reste dépositaire de la mémoire de son lieu, témoin de son regard, témoin dans sa chair, témoin dans son âme. D'autre part il est forcément obligé de dépasser son lieu dans sa quête obligée de l'humain, de l'universalité (Victor, 2007: 320).

Dans cette logique, si le concept de littérature nationale enferme les écrivains dans des identités étouffantes, car souvent stéréotypées, l'accès à l'universel passe tout de même par la recherche d'une forme d'authenticité qui n'est pas seulement individuelle, mais aussi collective, puisqu'il s'agit d'être "le dépositaire de la mémoire de son lieu". En fait, les deux aspects - l'universel et le particulier - sont interdépendants, dans la mesure où le créateur ne semble pouvoir affronter la mondialisation et ne peut l'affronter qu'en poursuivant simultanément une double quête du local et de l'universel. Les termes employés par Alain Mabanckou et Gary Victor - le "local", le "lieu" - montrent que ces auteurs ne veulent pas se prononcer sur ce qui définit l'identité et sont bien conscients du fait qu'elle ne saurait se résumer à une détermination nationale, régionale ou culturelle. La notion de littérature nationale telle qu'elle était admise dans la première moitié du $X X^{e}$ siècle est donc remplacée par un rapport plus complexe - mais non moins nécessaire - entre création artistique, identité individuelle et identité collective.

Envisagée de la sorte, la dialectique de l'universel et du particulier se prête alors à un certain nombre de revendications de nature politique. La première est une contestation de l'idée selon laquelle la littérature française occuperait une place prééminente dans le champ littéraire de langue française. Dans sa contribution à Pour une littérature-monde, intitulée "Mort d'une certaine idée", Jean Rouaud conclut au déclin de la littérature française contemporaine et explique ce déclin par celui de la France elle-même, qui, à la suite de la défaite de 1940 et de la Seconde Guerre mondiale, aurait peu à peu cessé de croire en la 
fiction de sa vocation universelle, qui la soutenait jusque là. La décadence de la littérature française se serait manifestée par un renoncement à dire le monde tel qu'il est au profit d'une écriture de plus en plus intimiste et de plus en plus formaliste, dont les courants du Nouveau Roman et de l'Oulipo constitueraient les exemples les plus frappants. II va de soi que cette thèse, qui réactive à sa manière la vieille opposition du vitalisme et du symbolisme (Wilfert-Portal, 2008), résiste mal à un examen critique, ne serait-ce que parce que l'idée de déclin est moins une réalité qu'un "mythe politique", pour reprendre une expression de I'historien Raoul Girardet ${ }^{3}$. Mais ce qui est significatif, surtout, c'est que Jean Rouaud, après avoir dressé un bilan accablant, envisage tout de même une issue heureuse à la crise, une délivrance venue non de la littérature écrite en France, mais de la littérature écrite en langue française aux quatre coins du monde:

II y eut une autre conséquence heureuse à cette disparition du pays, une clameur venue d'ailleurs, et qui nous fit tendre l'oreille au moment où nous étions au chevet du grand corps agonisant. [...] [N]ous avions peu à peu oublié que la langue avait fait souche sur les cinq continents [...]. Et que désormais déliée de son pacte avec la nation, libérée de l'étreinte de la source-mère, devenue autonome, [...] elle avait de nouveau à proposer, vue d'Afrique, d'Asie ou des Caraïbes, de Chine ou d'Iran, d'Amérique du Nord ou du Vietnam, son interprétation du monde (Rouaud, 2007: 21).

Si l'on suit ce raisonnement, l'écriture aurait vocation à parler du monde tel qu'il est, et non à se replier sur elle-même, de sorte que, face à la démission des écrivains français, ce seraient les auteurs dits francophones qui auraient pris le relais et se montreraient les plus fidèles à la véritable mission de la littérature, qui consisterait à produire des œuvres de portée universelle en proposant une "interprétation du monde". Ce thème d'une fonction de la littérature ne laisse pas de surprendre. Non seulement son caractère prescriptif, qui correspond sans doute au genre littéraire du manifeste (Forsdick, 2010: 9), s'accorde mal avec la revendication de liberté exprimée par la plupart des contributeurs, mais en plus il ne tient pas compte du fait que les textes possèdent une histoire culturelle et que la dimension universelle d'une œuvre - si tant est que l'expression ait un sens, ce qui ne va pas de soi dépend avant tout de la manière dont cette œuvre est lue, reçue et commentée un peu partout dans le monde. Jean Rouaud n'envisage pas la "littérature-monde" comme le résultat

\footnotetext{
${ }^{3}$ Raoul Girardet définit le mythe politique comme un "système de croyance cohérent et complet", un "ensemble structur[al] d'une réelle homogénéité et d'une constante spécificité" (Girardet, 1986: 12) dépassant les clivages partisans et suscitant l'adhésion d'une large partie de l'opinion. II identifie quatre grands mythes politiques structurant la vie politique française des $X I X^{\mathrm{e}}$ et $X X^{\mathrm{e}}$ siècles: l'âge d'or, la conspiration, le sauveur et l'unité. Mais la question de la décadence se trouve au cœur de son analyse: "[Au-delà] de l'extrême diversité [des] expressions [du mythe], il reste en fait la possibilité de constater encore une fois la permanence d'une structure, la présence solidement affirmée de la cohérence d'une logique. Logique [...] qui n'est en fin de compte rien d'autre que celle de la décadence“" (Girardet, 1986: 104-105).
} 
de la circulation, de la traduction et de la réception des textes, ainsi qu'a pu le faire notamment David Damrosch dans What Is World Literature ? $^{4}$, mais comme un ensemble de propriétés intrinsèques, définies de manière surtout négative (le refus de l'intellectualisme jargonnant, du formalisme gratuit, de l'intimisme nombriliste), que possèderaient certaines œuvres.

Quoi qu'il en soit, l'idée selon laquelle la France contemporaine ne serait plus un lieu propice à la production d'une littérature donnant accès à l'universel imprègne bel et bien Pour une littérature-monde. Or parallèlement à la critique de la France se développent des discours de valorisation d'autres identités, qui lient création littéraire et appartenance culturelle. C'est ainsi que Gary Victor, par exemple, fait l'éloge d'une identité "caribéenne" qu'il estime ouverte à la diversité du monde:

Chez nous, nous étions déjà ouverts au monde, en quête de tout ce qui se produisait comme littérature dans le monde entier. Certainement, comme l'a souligné maintes fois Edouard Glissant, parce que la Caraïbe est incontestablement le lieu qui instruit le mieux l'esprit à la diversité, à la rencontre, au partage (Victor, 2007: 316-317).

Les lecteurs originaires de la Caraïbe seraient curieux de toute la littérature du monde et cette remarquable ouverture d'esprit expliquerait pourquoi les écrivains issus de la région, qui sont avant tout des lecteurs, possèderaient une aptitude particulière à la recherche de l'universel, défini comme le refus des carcans et l'exploration de pistes à la fois originales et personnelles:

Nous avons appris à être à l'écoute de la mer et des alizés et comme la mer et son souffle sont l'âme de la planète, nous avons compris que le souffle de la création n'avait pas de frontières. [...] Heureusement qu'en Haïti les écrivains se sont dégagés [...] de ces carcans [il s'agit des règles et des canons imposés par les tenants d'une littérature nationale], libres maintenant d'explorer chacun les pistes beaucoup plus adaptées à leurs sensibilités propres (Victor, 2007: 317).

L'accès à l'universel est alors inséparable d'un discours de valorisation identitaire, dans un contexte d'affrontement entre le Nord et le Sud:

\footnotetext{
${ }^{4}$ David Damrosch insiste en effet sur les processus de circulation et de traduction des textes, qui font de la littérature monde un système en constante évolution, fondé sur des échanges culturels incessants: "[...] works of world literature take on a new life as they move into the world at large, and to understand this new life we need to look closely at the ways the work becomes reframed in its translations and in its new cultural contexts" (Damrosch, 2003: 24).
} 
Le Nord est venu chercher l'or chez nous mais uniquement ce métal qui a fait couler tant de sang et qui a permis de renforcer un monumental empire qui impose souvent de manière arrogante son mode de pensée et de vie. Mais dans la Caraïbe, nous avons appris à être autre chose. Chercheur d'or mais chercheur d'essentiel. Toujours en quête de l'humain (Victor, 2007: 317).

La dialectique du local et de l'universel se double ainsi d'une forme de contestation des rapports géopolitiques entre le Nord et le Sud. Or il est curieux de noter que ce discours de valorisation de l'identité "caribéenne" utilise les mêmes arguments que le discours de promotion de l'identité française que l'on rencontre chez Gide ou Valéry. L'accès à l'universel, dans les deux cas, dépend en effet d'une expérience de la diversité jugée sans équivalent, car propre à un espace qui possèderait une véritable vocation au cosmopolitisme. L'idée selon laquelle "la Caraïbe est incontestablement le lieu qui instruit le mieux l'esprit à la diversité" est une construction idéologique, au même titre que la représentation d'une France riche de la diversité de ses provinces et capable de ce fait d'effectuer la synthèse d'influences culturelles variées; elle est destinée à lutter contre les ravages de l'impérialisme culturel français.

Une seconde revendication de nature politique, présente dans Pour une littératuremonde et mettant en jeu la notion de littérature nationale, concerne la création de "lectorat[s] nationa[ux] à venir". Dans sa contribution au recueil, Nimrod, après avoir critiqué la notion d'authenticité, affirme que l'on écrit cependant toujours pour une nation:

Quelle que soit la race d'écrivains dont on procède, on n'écrit jamais que pour un pays, une nation, quelle que soit la portée universelle de notre œuvre. La littérature française n'est grande que parce que, à tort ou à raison, elle incarne la France. On écrit donc pour un pays, même si, comme chez Beckett, il n'est jamais fait mention d'appartenance nationale (Nimrod, 2007: 227).

Au premier abord, cette déclaration peut surprendre. Pourtant, la contradiction n'en est pas une si l'on admet que le refus de l'idée d'authenticité, chez Nimrod, n'est autre que le refus de la vision occidentale stéréotypée d'une Afrique villageoise, pétrifiée dans des traditions immuables. En revanche, il existerait bel et bien, selon lui, un lien étroit entre l'écrivain et sa nation, mais ce lien n'aurait rien à voir avec une simple préservation du patrimoine. La situation marginale des auteurs africains dans le champ littéraire de langue française s'expliquerait par la situation de domination néocoloniale que subissent encore la plupart des anciennes colonies de la France: "[...] la littérature africaine d'expression française serait déjà une grande émancipée si les pays africains existaient vraiment" 
(Nimrod, 2007: 227-228) ${ }^{5}$. C'est pourquoi les auteurs africains ne s'adressent pas à des lectorats nationaux déjà existants, mais à des lectorats nationaux à venir, qui ne verront le jour que lorsque la sujétion néocoloniale et la domination économique auront pris fin: "[...] nous écrivons pour des pays qui n'existent pas encore, nous écrivons une littérature pour un lectorat national à venir" (Nimrod, 2007: 228). En attendant, il ne s'agit pas de cultiver des traditions mythifiées, mais de produire des œuvres pour le public africain réel, c'est-à-dire pour un public moderne, majoritairement urbain, très éloigné des fantasmes primitivistes de l'Occident et ayant, depuis la colonisation, fait l'expérience d'une profonde hybridité culturelle:

Nous sommes des hybrides. [...] Une littérature qui traduit une semblable vérité gagne, à tous les coups, son ticket pour l'universel - terme dont on usait avant l'invention de "littérature-monde". La littérature ne parle jamais que de l'autre et de la langue de l'autre, cet autre qu'elle invente et qui s'invente dans le procès des vocables (Nimrod, 2007: 233).

Cette définition de la littérature comme parole de l'autre, qui n'est à aucun moment discutée alors qu'elle n'a pourtant rien d'une évidence, permet à Nimrod de formuler à sa manière la dialectique du local et de l'universel. Selon lui, pour un écrivain africain, accéder à l'universel implique de s'adresser aux lecteurs africains tels qu'ils existent vraiment, et non tels qu'on se les représente en Occident; il s'agit d'écrire pour des lecteurs qui, parce qu'ils vivent une situation de dualité culturelle, sont particulièrement disposés à accueillir une littérature hybride, donc tendant à l'universel, et sont les premiers destinataires de la révolution linguistique, esthétique et littéraire en train de s'accomplir en Afrique, sous le signe du mélange: "La littérature africaine d'expression française a inventé une [...] nouvelle manière d'écrire le français. Elle a fomenté sa révolution, et l'a réussie" (Nimrod, 2007: 229). Pour illustrer son propos, il cite l'exemple d'Ahmadou Kourouma, qui a su "plier" le français à la syntaxe de sa langue maternelle, le malinké (Nimrod, 2007: 231), créant ainsi un style nouveau, inédit dans l'histoire littéraire. Afin de renouveler la littérature de langue française, les auteurs africains doivent donc cesser de refléter les représentations stéréotypées de l'Occident et écrire pour des "lectorats nationaux" encore à naître.

Dans Pour une littérature-monde, la dialectique de l'universel et du particulier, qui servait au début du $X X^{e}$ siècle à construire la notion de littérature nationale, est donc à la fois

\footnotetext{
${ }^{5}$ Depuis une dizaine d'années, les ravages de la politique néocoloniale menée par la France en Afrique commencent à être régulièrement dénoncés (Verschave, 1998), mais la mythologie coloniale, comme l'a notamment montré Catherine Coquio, ne disparaît pas pour autant et continue d'être exploitée à des fins électoralistes (Coquio, 2008).
} 
reprise et détournée, puisqu'elle est utilisée pour contester ce concept au nom de la complexité des identités. L'idée selon laquelle la fidélité à soi-même, au "local", au "lieu" dont on est issu ou que l'on porte en soi serait le meilleur moyen d'accéder à l'universel reste cependant bien ancrée, même s'il convient de rappeler que le recueil se caractérise avant tout par sa diversité et que certains contributeurs refusent catégoriquement toute forme d'assignation identitaire. La défense d'une "littérature-monde en français", qui se veut une rupture avec la tradition littéraire française, reprend ainsi, tout en les renouvelant et en les modifiant, certains modes de pensée présents depuis longtemps dans cette tradition. Les notions de local et d'universel, aussi floues soient-elles, sont employées dans le cadre d'une stratégie de lutte contre les ravages de l'impérialisme culturel français, même si, paradoxalement, le manifeste et le livre, publiés respectivement dans l'un des plus grands quotidiens français et chez l'un des principaux éditeurs parisiens, utilisent les canaux de diffusion les mieux établis et ne contribuent de ce point de vue que modestement à la remise en cause de la domination parisienne sur le champ littéraire de langue française. 


\section{Bibliographie}

APPADURAI, Arjun (2005). Après le colonialisme. Les conséquences culturelles de la globalisation. Paris: Payot \& Rivages ( $1^{\text {ere }}$ édition, en anglais: 1996).

BARRÈS, Maurice (1994). Les Déracinés. In: Maurice Barrès. Romans et voyages. Paris: Robert Laffont, Collection Bouquins (1 ${ }^{\text {ere }}$ édition des Déracinés: 1897).

CASANOVA, Pascale (1999). La République mondiale des lettres. Paris: Seuil.

CÉLESTIN, Roger \& DALMOLIN, Eliane (éds.) (2010). Littérature-monde: New Wave or New Hype?. In: Contemporary French and Francophone Studies, vol. 14, n91, janvier 2010.

COQUIO, Catherine (2008). "Retours du colonial?". In: Catherine Coquio (dir.). Retours du colonial? Disculpation et réhabilitation de l'histoire coloniale. Nantes: L'Atalante, pp. 9-43.

DAMROSCH, David (2003). What Is World Literature? Princeton \& Oxford: Princeton University Press.

DE TOLEDO, Camille (2008). Visiter le Flurkistan ou les illusions de la littérature monde. Paris: Presses Universitaires de France.

DI MÉO, Nicolas (2009). Le Cosmopolitisme dans la littérature française de Paul Bourget à Marguerite Yourcenar. Genève: Droz.

FORSDICK, Charles (2010). "From 'Littérature voyageuse' to 'Littérature-monde': the Manifesto in Context". In: Contemporary French and Francophone Studies, vol. 14, nㅇ, janvier 2010, pp. 9-17.

GIDE, André (1999). "Nationalisme et littérature". In: André Gide. Essais critiques. Paris: Gallimard, Bibliothèque de la Pléiade ( $1{ }^{\text {ère }}$ édition de "Nationalisme et littérature": 1909).

GIDE, André (2001). Retour de l'U.R.S.S. In: André Gide. Souvenirs et voyages. Paris: Gallimard, Bibliothèque de la Pléiade ( ${ }^{\text {ère }}$ édition de Retour de l'U.R.S.S.: 1936).

GIRARDET, Raoul (1986). Mythes et mythologies politiques. Paris: Seuil.

HUSTON, Nancy (2007). "Traduttore non è traditore". In: Jean Rouaud, Michel Le Bris (dirs.). Pour une littérature-monde. Paris: Gallimard, pp. 151-160.

MABANCKOU, Alain (2007). "Le chant de l'oiseau migrateur". In: Jean Rouaud, Michel Le Bris (dirs.). Pour une littérature-monde. Paris: Gallimard, pp. 55-66.

MOURA, Jean-Marc (2010). "French Language Writing and the Francophone Literary System". In: Contemporary French and Francophone Studies, vol. 14, n9, janvier 2010, pp. 29 -38.

NIMROD (2007). "La nouvelle chose française: pour une littérature décolonisée". In: Jean Rouaud, Michel Le Bris (dirs.). Pour une littérature-monde. Paris: Gallimard, pp. 217-235.

ROUAUD, Jean (2007). "Mort d'une certaine idée”. In: Jean Rouaud, Michel Le Bris (dirs.). Pour une littérature-monde. Paris: Gallimard, pp. 7-22.

THIESSE, Anne-Marie (1999). La Création des identités nationales. Paris: Seuil.

VALÉRY, Paul (1960). Regards sur le monde actuel. In: Paul Valéry. CEuvres. Paris: Gallimard, Bibliothèque de la Pléiade, vol. II.

VERSCHAVE, François-Xavier (1998). La Françafrique. Paris: Stock.

VICTOR, Gary (2007). "Littérature-monde ou liberté d'être". In: Jean Rouaud, Michel Le Bris (dirs.). Pour une littérature-monde. Paris: Gallimard, pp. 315-320.

WABERI, Abdourahman A. (2007). "Ecrivains en position d'entraver". In: Jean Rouaud, Michel Le Bris (dirs.). Pour une littérature-monde. Paris: Gallimard, pp. 67-75.

WILFERT-PORTAL, Blaise (2008). "La Littérature française dans la mondialisation". [compte rendu de Pour une littérature-monde] [en ligne] In: La Vie des idées.fr. [consulté le 31/01/2010] <URL: http://www.laviedesidees.fr/La-litterature-francaise-dans-la.html> 